\title{
Vorwort zur 3. Auflage
}

Die ersten beiden Auflagen dieses Buches erschienen in der Alleinautorenschaft von Klaus Schredelseker, der für die vorliegende dritte Auflage mit Felix Holzmeister und Jürgen Huber zwei seiner Schüler eingeladen hat, das Buch um Beiträge aus ihrem Forschungsbereich zu erweitern. Neben einigen redaktionellen Änderungen wurde das Buch etwas gekürzt, ohne jedoch die Kerninhalte und die typische Herangehensweise zu verändern (informationstheoretischer Zugang). Neu hinzugekommen sind insbesondere Ansätze und Beispiele aus der Behavioral Finance sowie ein Abschnitt über Nutzenfunktionen um die Prospect Theory. Die zweite wesentliche Erweiterung stellen Experimente zur Nützlichkeit von Informationen in Märkten dar. Das in der ersten Auflage enthaltene agentenbasierte Modell wurde in der zweiten Auflage deutlich erweitert und zusätzlich um ein von Hauser/Kaempff entwickeltes evolutorisches Modell ergänzt. In der dritten Auflage erweitern wir diesen Methodenmix nun um Ergebnisse aus Laborexperimenten, in denen die Teilnehmer in einer vollständig kontrollierbaren Umgebung eines von uns programmierten Marktes Handelsentscheidungen treffen. Die Ergebnisse bestätigen dabei die Erkenntnisse aus den agentenbasierten Modellen; diesmal aber mit echten Agenten anstelle der Algorithmen.

Alle Tabellen im Buch basieren, sofern nicht anders gekennzeichnet, auf eigenen Daten bzw. Berechnungen. Alle Abbildungen im Buch sind, sofern nicht anders gekennzeichnet, eigene Darstellungen.

Innsbruck

Februar 2019

Felix Holzmeister, Jürgen Huber und Klaus Schredelseker 